\title{
ANALYSIS OF THE BOUNDARY SYMBOL FOR THE TWO-PHASE NAVIER-STOKES EQUATIONS WITH SURFACE TENSION
}

\author{
JAN PRÜSS \\ Institut für Mathematik \\ Martin-Luther-Universität Halle-Wittenberg \\ D-60120 Halle, Germany \\ E-mail: jan.pruess@mathematik.uni-halle.de \\ GIERI SIMONETT \\ Department of Mathematics, Vanderbilt University \\ Nashville, TN, U.S.A. \\ E-mail: gieri.simonett@vanderbilt.edu
}

\begin{abstract}
The two-phase free boundary value problem for the Navier-Stokes system is considered in a situation where the initial interface is close to a halfplane. We extract the boundary symbol which is crucial for the dynamics of the free boundary and present an analysis of this symbol. Of particular interest are its singularities and zeros which lead to refined mapping properties of the corresponding operator.
\end{abstract}

1. Introduction. In this paper we consider a free boundary problem that describes the motion of two viscous, incompressible, capillary Newtonian fluids in $\mathbb{R}^{3}$. The fluids are separated by an interface that is unknown and has to be determined as part of the problem.

Let $\Gamma_{0} \subset \Omega$ be a given surface which bounds a region $\Omega_{1}(0)$ occupied by a viscous incompressible fluid, fluid 1 , and let $\Omega_{2}(0)$ be the complement of the closure of $\Omega_{1}(0)$ in $\mathbb{R}^{3}$, corresponding to the region occupied by a second incompressible viscous fluid, fluid 2. We assume that the two fluids are immiscible. Let $\Gamma(t)$ denote the position of $\Gamma_{0}$ at time $t$. Thus, $\Gamma(t)$ is a sharp interface which separates the fluids occupying the regions $\Omega_{1}(t)$ and $\Omega_{2}(t)$, respectively. We denote the normal field on $\Gamma(t)$, pointing from $\Omega_{1}(t)$

2000 Mathematics Subject Classification: 35R35, 35Q30, 76D45, 76T10.

Key words and phrases: Navier-Stokes equations, surface tension, boundary symbol, fundamental solution.

The paper is in final form and no version of it will be published elsewhere. 
into $\Omega_{2}(t)$, by $\nu(t, \cdot)$. Moreover, we denote by $V(t, \cdot)$ and $\kappa(t, \cdot)$ the normal velocity and the mean curvature of $\Gamma(t)$ with respect to $\nu(t, \cdot)$, respectively. The motion of the fluids is governed by the following system of equations for $i=1,2$ :

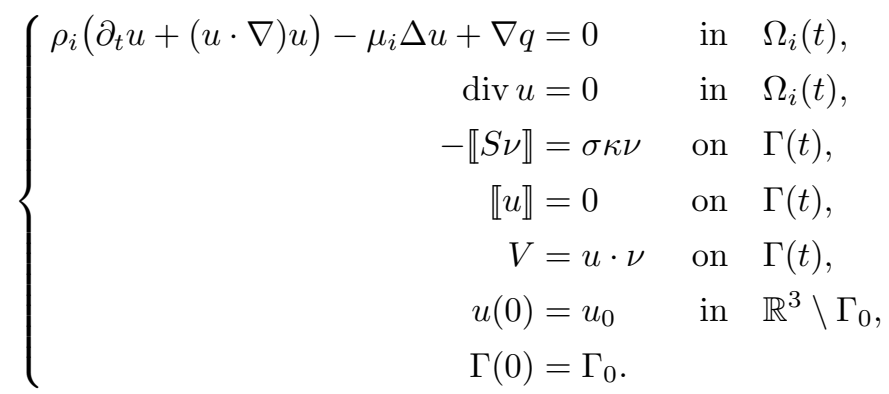

Here, $S$ is the stress tensor defined by

$$
S=\mu_{i}\left(\nabla u+(\nabla u)^{\top}\right)-q I \quad \text { in } \quad \Omega_{i}(t),
$$

and

$$
\llbracket v \rrbracket=\left.\left(v_{\left.\right|_{\Omega_{2}(t)}}-v_{\left.\right|_{\Omega_{1}(t)}}\right)\right|_{\Gamma(t)}
$$

denotes the jump of the quantity $v$, defined on the respective domains $\Omega_{i}(t)$, across the interface $\Gamma(t)$.

Given are the initial position $\Gamma_{0}$ of the interface, and the initial velocity $u_{0}: \mathbb{R}^{3} \backslash \Gamma_{0}$ $\rightarrow \mathbb{R}^{3}$. The unknowns are the velocity field $u(t, \cdot): \mathbb{R}^{3} \backslash \Gamma(t) \rightarrow \mathbb{R}^{3}$, the pressure field $q(t, \cdot): \mathbb{R}^{3} \backslash \Gamma(t) \rightarrow \mathbb{R}$, and the free boundary $\Gamma(t)$. The constants $\rho_{i}>0$ and $\mu_{i}>0$ denote the densities and the viscosities of the respective fluids, and the constant $\sigma$ stands for the surface tension. System (1.1) comprises the two-phase Navier-Stokes equations with surface tension.

In order to economize our notation, we set

$$
\rho=\rho_{1} \chi_{\Omega_{1}(t)}+\rho_{2} \chi_{\Omega_{2}(t)}, \quad \mu=\mu_{1} \chi_{\Omega_{1}(t)}+\mu_{2} \chi_{\Omega_{2}(t)},
$$

where $\chi_{D}$ denotes the indicator function of the set $D$. With this convention system (1.1) can be recast as

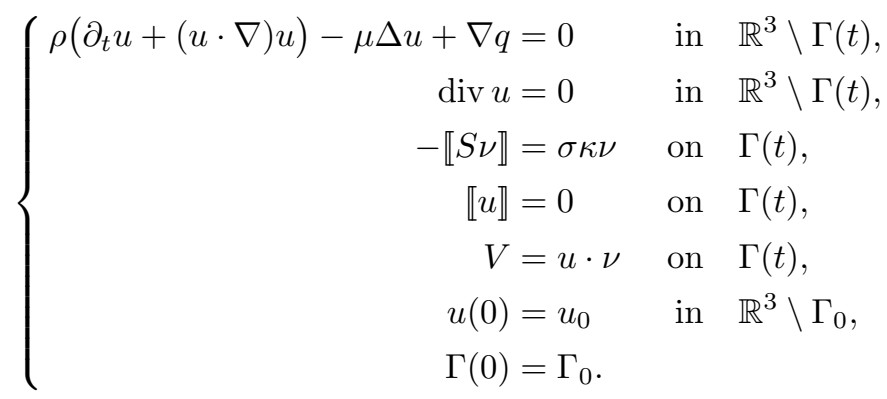

Existence and uniqueness of solutions for the corresponding one-phase problem, which is obtained from the two-phase problem by setting $u \equiv q \equiv 0$ on $\Omega_{2}(t)$ and discarding $\Omega_{2}(t)$, has been extensively studied in a long series of papers by Solonnikov, see for instance $[17,18,19,20,21,15]$ for the case $\sigma>0$. The authors in $[1,2,23,24,16]$ consider the motion of a layer of heavy, viscous, incompressible fluid in an ocean of infinite extend that 
is bounded below by a solid surface and above by a free surface which includes the effects of surface tension. The authors obtain local existence and uniqueness of solutions. Existence and uniqueness of solutions for the two-phase problem (1.2) is studied in $[4,5,6,22]$.

Here we are interested in the situation where $\Gamma_{0}$ is close to a plane, say $\mathbb{R}^{n}$ with $n \geq 2$, i.e. $\Gamma_{0}$ is a graph over $\mathbb{R}^{n}$ given by a function $h_{0}$ with $\left|\nabla h_{0}\right|_{\infty}$ small. Then it is natural to transform the problem to a flat fixed interface, and solve the resulting quasilinear evolution problem. The general situation where $\Gamma_{0}$ is a closed compact manifold can then be treated by a localization procedure. This will be carried out in a forthcoming paper. Our basic well-posedness and regularity result for the geometry considered in this paper reads as follows.

THEOREM 1.1. Fix $p>n+3$ and let $h_{0} \in W_{p}^{3-2 / p}\left(\mathbb{R}^{n}\right), u_{0} \in W_{p}^{2-2 / p}\left(\mathbb{R}^{n+1} \backslash \Gamma_{0}\right)$ be given. Assume that the compatibility conditions

$$
\operatorname{div} u_{0}=0 \text { on } \mathbb{R}^{n+1} \backslash \Gamma_{0}, \quad \llbracket D_{0} \nu-\left(\nu \cdot D_{0} \nu\right) \nu \rrbracket=0, \quad \llbracket u_{0} \rrbracket=0 \text { on } \Gamma_{0},
$$

are satisfied, where $D_{0}=\left(\nabla u_{0}+\left(\nabla u_{0}\right)^{\top}\right)$. Then there exists $\eta>0$ such that for $\left|\nabla h_{0}\right|_{\infty}<$ $\eta$ there is $t_{0}=t_{0}\left(u_{0}, h_{0}\right)>0$ and a unique classical solution $(u, q, \Gamma)$ of problem (1.2) on $\left(0, t_{0}\right)$. In addition, $\Gamma(t)$ is a graph over $\mathbb{R}^{n}$ given by a function $h(t)$,

$$
\mathcal{M}=\bigcup_{t \in\left(0, t_{0}\right)}\{t\} \times \Gamma(t)
$$

is a real analytic manifold, and with

$$
\Omega:=\left\{(t, x, y): t \in\left(0, t_{0}\right), x \in \mathbb{R}^{n}, y \neq h(t, x)\right\},
$$

the function $(u, q): \Omega \rightarrow \mathbb{R}^{n+2}$ is real analytic.

Due to the restriction $p>n+3$, we obtain

$$
h \in C\left(J ; B U C^{2}\left(\mathbb{R}^{n}\right)\right), \quad \partial_{t} h \in C\left(J ; B U C^{1}\left(\mathbb{R}^{n}\right)\right),
$$

where $J=\left[0, t_{0}\right]$. In particular, the normal of $\Omega_{1}(t)$, the normal velocity of $\Gamma(t)$ and the mean curvature of $\Gamma(t)$ are well-defined and continuous, so that (1.2) makes sense pointwise. For $u$ we obtain

$$
u \in B U C\left(J \times \mathbb{R}^{n}\right), \quad \nabla u \in B U C(\Omega),
$$

and $(u, q, h)$ depend continuously on the data $u_{0}$ and $h_{0}$. Also interesting is the fact that the surface pressure jump satisfies $\llbracket q \rrbracket \in B U C(\mathcal{M})$ and is real analytic as well.

In this paper we are interested in the boundary symbol $s(\lambda, \xi)$ of this problem, which determines the linearized dynamics of the interface. Here $\lambda$ resp. $\xi$ denote the covariables of time $t$ resp. $x$, the tangential variable on the flattened interface. We indicate how to derive this symbol and present a detailed analysis of its properties.

The plan for this paper is as follows. Section 2 contains the transformation of the problem to a half-space and the determination of the proper underlying linear problem. In Section 3 we extract the boundary symbol, and we study its basic mapping properties in Section 4. An analysis of the characteristic equation $s(\lambda, \xi)=0$ is given in Section 5 , and the refined mapping properties of the corresponding operator are presented in Sections 6 and 7 . 
2. Reduction to a flat interface and linearization. Suppose that $\Gamma(t)$ is a graph over $\mathbb{R}^{n}$, parametrized as

$$
\Gamma(t)=\left\{(x, h(t, x)): x \in \mathbb{R}^{n}\right\}, \quad t \in J:=[0, a] .
$$

Then we introduce the transformed variables

$$
\begin{aligned}
& v(t, x, y)=\left[\begin{array}{l}
u_{1}(t, x, h(t, x)+y) \\
\vdots \\
u_{n}(t, x, h(t, x)+y)
\end{array}\right], \\
& w(t, x, y)=u_{n+1}(t, x, h(t, x)+y), \\
& \pi(t, x, y)=q(t, x, h(t, x)+y),
\end{aligned}
$$

where $t \in J=[0, a], x \in \mathbb{R}^{n}, y \in \mathbb{R}, y \neq 0$. By means of this coordinate transform the interface $\Gamma(t)$ is $\mathbb{R}^{n}$, independently of $t$. The two-phase Navier-Stokes problem with surface tension then becomes the following quasilinear problem on the fixed domain $\dot{\mathbb{R}}^{n+1}:=\left\{(x, y) \in \mathbb{R}^{n} \times \mathbb{R}: y \neq 0\right\}$ for the new unknowns $u:=(v, w), \pi$ and $h$ :

$$
\left\{\begin{aligned}
\rho \partial_{t} u-\mu \Delta u+\nabla \pi & =F(u, \pi, h) & & \text { in } \quad \dot{\mathbb{R}}^{n+1}, \\
\operatorname{div} u & =F_{d}(u, h) & & \text { in } \quad \dot{\mathbb{R}}^{n+1}, \\
-\llbracket \mu \partial_{y} v \rrbracket-\llbracket \mu \nabla_{x} w \rrbracket & =G_{v}(u, \llbracket \pi \rrbracket, h) & & \text { on } \quad \mathbb{R}^{n}, \\
-2 \llbracket \mu \partial_{y} w \rrbracket+\llbracket \pi \rrbracket-\sigma \Delta_{x} h & =G_{w}(u, h) & & \text { on } \quad \mathbb{R}^{n}, \\
\llbracket u \rrbracket & =0 & & \text { on } \quad \mathbb{R}^{n}, \\
\partial_{t} h-\left.w\right|_{y=0} & =H(u, h) & & \text { on } \quad \mathbb{R}^{n}, \\
u(0) & =u_{0} & & \text { in } \quad \dot{\mathbb{R}}^{n+1}, \\
h(0) & =h_{0}, & &
\end{aligned}\right.
$$

for all $t>0$. More details on this transformation, on the nonlinear right hand sides, as well as for the proof of Theorem 1.1 can be found in the forthcoming paper [13].

Thus the linearization of (2.1) at $h=0$ leads to the linear inhomogeneous problem

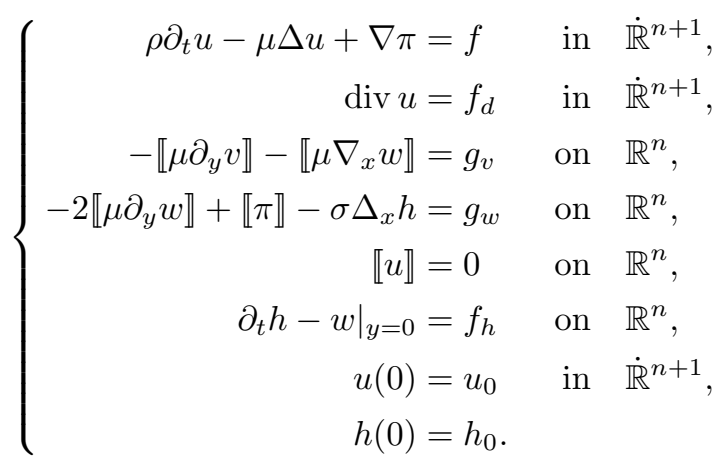

We are interested in the regularity class

$$
u \in H_{p}^{1}\left(J ; L_{p}\left(\mathbb{R}^{n+1}\right)\right) \cap L_{p}\left(J ; H_{p}^{2}\left(\dot{\mathbb{R}}^{n+1}\right)\right), \quad \pi \in L_{p}\left(J ; \dot{H}_{p}^{1}\left(\dot{\mathbb{R}}^{n+1}\right)\right),
$$

where $J=[0, a]$ means a finite time interval. In the following, $W_{p}^{m}$ denote as usual the Sobolev spaces if $m \in \mathbb{Z}$. For non-integer $s, W_{p}^{s}$ are the Sobolev-Slobodeckii spaces, and 
$H_{p}^{s}$ the Bessel-potential spaces. For $K \in\{H, W\}$, by $\dot{K}_{p}^{s}$ we mean the homogeneous version of $K_{p}^{s}$. Note that $H_{p}^{s}=W_{p}^{s}$ for integer values of $s$, but that in general these spaces are different.

If we assume a solution in the class (2.3), then for the right hand sides $f$ and $f_{d}$ we necessarily must have $f \in L_{p}\left(J \times \mathbb{R}^{n+1}\right)$ and

$$
f_{d} \in H_{p}^{1}\left(J ; \dot{H}_{p}^{-1}\left(\mathbb{R}^{n+1}\right)\right) \cap L_{p}\left(J ; H_{p}^{1}\left(\dot{\mathbb{R}}^{n+1}\right)\right),
$$

since the operator div maps $L_{p}$ into $H_{p}^{-1}$. By trace theory we necessarily have $u_{0} \in$ $W_{p}^{2-2 / p}\left(\dot{\mathbb{R}}^{n+1}\right)$, and the lateral trace of $u$ belongs to

$$
Y_{0}:=W_{p}^{1-1 / 2 p}\left(J ; L_{p}\left(\mathbb{R}^{n}\right)\right) \cap L_{p}\left(J ; W_{p}^{2-1 / p}\left(\mathbb{R}^{n}\right)\right),
$$

and that of $\nabla u$ to

$$
Y_{1}:=W_{p}^{1 / 2-1 / 2 p}\left(J ; L_{p}\left(\mathbb{R}^{n}\right)\right) \cap L_{p}\left(J ; W_{p}^{1-1 / p}\left(\mathbb{R}^{n}\right)\right) .
$$

Therefore $g_{v} \in Y_{1}$, and if in addition

$$
\llbracket \pi \rrbracket \in W_{p}^{1 / 2-1 / 2 p}\left(J ; L_{p}\left(\mathbb{R}^{n}\right)\right) \cap L_{p}\left(J ; W_{p}^{1-1 / p}\left(\mathbb{R}^{n}\right)\right),
$$

then we also have that $g_{w} \in Y_{1}$.

Concerning the regularity of the height function $h$ we note that the equation for $h$ lives in the trace space $Y_{0}$, hence naturally $h$ should belong to

$$
h \in W_{p}^{2-1 / 2 p}\left(J ; L_{p}\left(\mathbb{R}^{n}\right)\right) \cap H_{p}^{1}\left(J ; W_{p}^{2-1 / p}\left(\mathbb{R}^{n}\right)\right) .
$$

On the other hand, the equation for the normal component of the normal stress lives in $Y_{1}$, and contains the term $\Delta_{x} h$, hence $h$ should also belong to $L_{p}\left(J ; W_{p}^{3-1 / p}\left(\mathbb{R}^{n}\right)\right)$. These considerations lead to the following natural space for $h$

$$
h \in W_{p}^{2-1 / 2 p}\left(J ; L_{p}\left(\mathbb{R}^{n}\right)\right) \cap H_{p}^{1}\left(J ; W_{p}^{2-1 / p}\left(\mathbb{R}^{n}\right)\right) \cap L_{p}\left(J ; W_{p}^{3-1 / p}\left(\mathbb{R}^{n}\right)\right) .
$$

This then implies $f_{h} \in Y_{0}$, as well as $h_{0} \in W_{p}^{3-2 / p}\left(\mathbb{R}^{n}\right)$ by trace theory. Our next theorem states that in this setting, problem (2.2) admits maximal regularity; the described regularities of the data are also sufficient. In particular, the solution map defines an isomorphism between this space of data and the solution space defined above.

THEOREM 2.1. Let $1<p<\infty$ be fixed, $p \neq 3 / 2,3$, and assume that $\rho_{j}$ and $\mu_{j}$ are positive constants for $j=1,2$, and set $J=[0, a]$. Then the instationary Stokes problem with free boundary (2.2) admits a unique solution $(u, \pi, h)$ with regularity

$$
\begin{gathered}
u \in H_{p}^{1}\left(J ; L_{p}\left(\mathbb{R}^{n+1}\right)\right) \cap L_{p}\left(J ; H_{p}^{2}\left(\dot{\mathbb{R}}^{n+1}\right)\right), \quad \pi \in L_{p}\left(J ; \dot{H}_{p}^{1}\left(\dot{\mathbb{R}}^{n+1}\right)\right), \\
\llbracket \pi \rrbracket \in W_{p}^{1 / 2-1 / 2 p}\left(J ; L_{p}\left(\mathbb{R}^{n}\right)\right) \cap L_{p}\left(J ; W_{p}^{1-1 / p}\left(\mathbb{R}^{n}\right)\right),
\end{gathered}
$$

and

$$
h \in W_{p}^{2-1 / 2 p}\left(J ; L_{p}\left(\mathbb{R}^{n}\right)\right) \cap H_{p}^{1}\left(J ; W_{p}^{2-1 / p}\left(\mathbb{R}^{n}\right)\right) \cap L_{p}\left(J ; W_{p}^{3-1 / p}\left(\mathbb{R}^{n}\right)\right)
$$

if and only if the data $\left(f, f_{d}, g, f_{h}, u_{0}, h_{0}\right)$ satisfy the following regularity and compatibility conditions:

(a) $\left.f \in L_{p}\left(J \times \mathbb{R}^{n+1}\right)\right)$,

(b) $f_{d} \in H_{p}^{1}\left(J ; \dot{H}_{p}^{-1}\left(\mathbb{R}^{n+1}\right)\right) \cap L_{p}\left(J ; H_{p}^{1}\left(\dot{\mathbb{R}}^{n+1}\right)\right)$, 
(c) $g=\left(g_{v}, g_{w}\right) \in W_{p}^{1 / 2-1 / 2 p}\left(J ; L_{p}\left(\mathbb{R}^{n}\right)\right) \cap L_{p}\left(J ; W_{p}^{1-1 / p}\left(\mathbb{R}^{n}\right)\right)$,

(d) $f_{h} \in W_{p}^{1-1 / 2 p}\left(J ; L_{p}\left(\mathbb{R}^{n}\right)\right) \cap L_{p}\left(J ; W_{p}^{2-1 / p}\left(\mathbb{R}^{n}\right)\right)$,

(e) $u_{0} \in W_{p}^{2-2 / p}\left(\dot{\mathbb{R}}^{n+1}\right), h_{0} \in W_{p}^{3-2 / p}\left(\mathbb{R}^{n}\right)$,

(f) $\operatorname{div} u_{0}=f_{\left.p\right|_{t=0}}$ in $\dot{\mathbb{R}}^{n+1}$ and $\llbracket u_{0} \rrbracket=0$ on $\mathbb{R}^{n}$ if $p>3 / 2$,

(g) $-\llbracket \mu \partial_{y} v_{0} \rrbracket-\llbracket \mu \nabla_{x} w_{0} \rrbracket=g_{\left.v\right|_{t=0}}$ on $\mathbb{R}^{n}$ if $p>3$.

The solution map $\left[\left(f, f_{d}, g, f_{h}, u_{0}, h_{0}\right) \mapsto(u, \pi, h)\right]$ is continuous between the corresponding spaces.

Below we discuss in more detail the arguments which lead to sufficiency for the regularity of $h$. For the complete proof of Theorem 2.1 we refer to the paper [13].

3. Extraction of the boundary symbol. The main ingredient for the extraction of the boundary symbol is the Dirichlet-to-Neumann operator for the (instationary) Stokes equations. To define the Dirichlet-to-Neumann operator we consider the following problem:

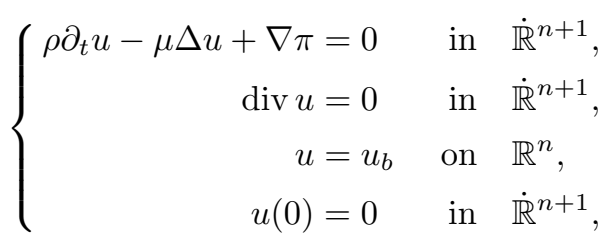

for $t>0$. We then define the Dirichlet-to-Neumann operator by means of

$$
\mathcal{D} N u_{b}=-\llbracket S \rrbracket e_{n+1}=-\llbracket \mu\left(\nabla u+(\nabla u)^{\top}\right) \rrbracket e_{n+1}+\llbracket \pi \rrbracket e_{n+1} .
$$

For this purpose it is convenient to split $u$ into $u=(v, w)$ as before, and $u_{b}$ into $u_{b}=$ $\left(v_{b}, w_{b}\right)$. Then we obtain

$$
\mathcal{D} N u_{b}=\left(-\llbracket \mu \partial_{y} v \rrbracket-\llbracket \mu \nabla_{x} w \rrbracket,-2 \llbracket \mu \partial_{y} w \rrbracket+\llbracket \pi \rrbracket\right) .
$$

We employ Laplace transform in $t$ and Fourier transform in the tangential variables $x \in \mathbb{R}^{n}$, to obtain the following boundary value problem for a system of ordinary differential equations on $\dot{\mathbb{R}}$ :

$$
\left\{\begin{aligned}
\omega^{2} v-\mu \partial_{y}^{2} v+i \xi \pi & =0, & & y \neq 0 \\
\omega^{2} w-\mu \partial_{y}^{2} w+\partial_{y} \pi & =0, & & y \neq 0 \\
(i \xi \mid v)+\partial_{y} w & =0, & & y=0 \\
v(0)=v_{b}, w(0) & =w_{b} . & &
\end{aligned}\right.
$$

Here we have set $\omega_{j}^{2}=\rho_{j} \lambda+\mu_{j}|\xi|^{2}, j=1,2$. Actually, we should have written something like $\hat{v}$, etc. to indicate that these functions have been transformed, but for simplicity we drop the hats. This system of equations is easily solved to give

$$
\left[\begin{array}{c}
v_{2} \\
w_{2} \\
\pi_{2}
\end{array}\right]=e^{-\omega_{2} y / \sqrt{\mu_{2}}}\left[\begin{array}{c}
a_{2} \\
\frac{\sqrt{\mu_{2}}}{\omega_{2}}\left(i \xi \mid a_{2}\right) \\
0
\end{array}\right]+\alpha_{2} e^{-|\xi| y}\left[\begin{array}{c}
-i \xi \\
|\xi| \\
\rho_{2} \lambda
\end{array}\right],
$$


for $y>0$, and

$$
\left[\begin{array}{c}
v_{1} \\
w_{1} \\
\pi_{1}
\end{array}\right]=e^{\omega_{1} y / \sqrt{\mu_{1}}}\left[\begin{array}{c}
a_{1} \\
-\frac{\sqrt{\mu_{1}}}{\omega_{1}}\left(i \xi \mid a_{1}\right) \\
0
\end{array}\right]+\alpha_{1} e^{|\xi| y}\left[\begin{array}{c}
-i \xi \\
-|\xi| \\
\rho_{1} \lambda
\end{array}\right]
$$

for $y<0$. Here $a_{i} \in \mathbb{R}^{n}$ and $\alpha_{i}$ have to be determined by the boundary conditions $v(0)=v_{b}$ and $w(0)=w_{b}$. We have

$$
a_{2}-i \xi \alpha_{2}=v_{b}=a_{1}-i \xi \alpha_{1}
$$

and

$$
\frac{\sqrt{\mu_{2}}}{\omega_{2}}\left(i \xi \mid a_{2}\right)+|\xi| \alpha_{2}=w_{b}=-\frac{\sqrt{\mu_{1}}}{\omega_{1}}\left(i \xi \mid a_{1}\right)-|\xi| \alpha_{1}
$$

This yields

$$
a_{j}=v_{b}+\alpha_{j} i \xi, \quad j=1,2,
$$

and

$$
\alpha_{2}=-\frac{\omega_{2}+|\xi| \sqrt{\mu_{2}}}{\rho_{2} \lambda|\xi|}\left(\sqrt{\mu_{2}}\left(v_{b} \mid i \xi\right)-\omega_{2} w_{b}\right)
$$

as well as

$$
\alpha_{1}=-\frac{\omega_{1}+|\xi| \sqrt{\mu_{1}}}{\rho_{1} \lambda|\xi|}\left(\sqrt{\mu_{1}}\left(v_{b} \mid i \xi\right)+\omega_{1} w_{b}\right) .
$$

We may now compute the symbol of the Dirichlet-to-Neumann operator to obtain

$$
\mathcal{D} N u_{b}=\left[\begin{array}{c}
\omega_{1} \sqrt{\mu_{1}} a_{1}+\omega_{2} \sqrt{\mu_{2}} a_{2}-\left(\alpha_{1} \mu_{1}+\alpha_{2} \mu_{2}\right)|\xi| i \xi-\llbracket \mu \rrbracket i \xi w_{b} \\
2 i\left(\mu_{2} a_{2}-\mu_{1} a_{1} \mid \xi\right)+2\left(\alpha_{2} \mu_{2}-\alpha_{1} \mu_{1}\right)|\xi|^{2}+\lambda\left(\alpha_{2} \rho_{2}-\alpha_{1} \rho_{1}\right)
\end{array}\right] .
$$

Simple algebraic manipulations then yield the following symbol for the Dirichlet-toNeumann operator

$$
\mathcal{D} N(\lambda, \xi)=\left[\begin{array}{cc}
\alpha+\beta \zeta \otimes \zeta & i \gamma \zeta \\
-i \gamma \zeta^{T} & \alpha+\delta
\end{array}\right]
$$

where $\zeta=\xi /|\xi|$ and

$$
\begin{gathered}
\alpha=\sqrt{\mu_{1}} \omega_{1}+\sqrt{\mu_{2}} \omega_{2}, \quad \beta=\left(\mu_{1}+\mu_{2}\right)|\xi| \\
\gamma=\left(\sqrt{\mu_{2}} \omega_{2}-\sqrt{\mu_{1}} \omega_{1}\right)-\llbracket \mu \rrbracket|\xi|, \quad \delta=\left(\omega_{1}^{2}+\omega_{2}^{2}\right) /|\xi|=\beta+\left(\rho_{1}+\rho_{2}\right) \lambda /|\xi| .
\end{gathered}
$$

Next we want to compute the symbol of the inverse of the Dirichlet-to-Neumann operator. Thus we have to solve the equation $\mathcal{D} N u_{b}=g$ on a symbolic level. We decompose as before $u_{b}=\left(v_{b}, w_{b}\right)$ and $g=\left(g_{v}, g_{w}\right)$. Then in transformed variables we have to solve the system

$$
\begin{aligned}
& \alpha v_{b}+\beta \zeta\left(v_{b} \mid \zeta\right)+i \gamma \zeta w_{b}=g_{v} \\
& -i \gamma\left(v_{b} \mid \zeta\right)+(\alpha+\delta) w_{b}=g_{w}
\end{aligned}
$$

This yields

$$
v_{b}=\alpha^{-1}\left[g_{v}-\zeta\left(\beta\left(v_{b} \mid \zeta\right)+i \gamma w_{b}\right)\right]
$$

thus it is sufficient to determine $\left(v_{b} \mid \zeta\right)$ and $w_{b}$. 
For $w_{b}$ and $\left(v_{b} \mid \zeta\right)$ we have the equations

$$
\begin{aligned}
& (\alpha+\beta)\left(v_{b} \mid \zeta\right)+i \gamma w_{b}=\left(g_{v} \mid \zeta\right), \\
& -i \gamma\left(v_{b} \mid \zeta\right)+(\alpha+\delta) w_{b}=g_{w},
\end{aligned}
$$

since $|\zeta|=1$. Solving this 2 -D system we obtain

$$
w_{b}=m^{-1}\left[i \gamma\left(g_{v} \mid \zeta\right)+(\alpha+\beta) g_{w}\right],
$$

and

$$
i\left(v_{b} \mid \zeta\right)=m^{-1}\left[(\alpha+\delta) i\left(g_{v} \mid \zeta\right)+\gamma g_{w}\right]
$$

where

$$
m=(\alpha+\beta)(\alpha+\delta)-\gamma^{2} .
$$

Since $\delta=\beta+\left(\rho_{1}+\rho_{2}\right) \lambda /|\xi|$ we obtain the following relation for $m$ :

$$
m=(\alpha+\beta)\left[\left(\rho_{1}+\rho_{2}\right) \lambda /|\xi|+4\left(\frac{1}{\eta_{1}}+\frac{1}{\eta_{2}}\right)^{-1}\right]=:(\alpha+\beta) n,
$$

where $\eta_{1}=\sqrt{\mu_{1}} \omega_{1}+\mu_{2}|\xi|$ and $\eta_{2}=\sqrt{\mu_{2}} \omega_{2}+\mu_{1}|\xi|$. This yields

$$
w_{b}=\frac{i \gamma}{(\alpha+\beta) n}\left(g_{v} \mid \zeta\right)+\frac{g_{w}}{n},
$$

and

$$
i\left(v_{b} \mid \zeta\right)=\frac{\left(\rho_{1}+\rho_{2}\right) \lambda /|\xi|}{(\alpha+\beta) n} i\left(g_{v} \mid \zeta\right)+\frac{1}{n}\left[i\left(g_{v} \mid \zeta\right)+\frac{\gamma}{\alpha+\beta} g_{w}\right] .
$$

To obtain the boundary symbol $s(\lambda, \xi)$ of the problem we now only need to set $g_{v}=0$, $g_{w}=-\sigma|\xi|^{2}$ and compute $w_{b}$ to obtain

$$
-w_{b}=\frac{\sigma|\xi|^{2}}{n}=\frac{\sigma|\xi|^{2}}{\left(\rho_{1}+\rho_{2}\right) \lambda /|\xi|+4 \eta_{1} \eta_{2} /\left(\eta_{1}+\eta_{2}\right)} .
$$

Inserting this into the transformed equation for $h$, i.e. $\lambda h-w_{b}=f_{h}$, we obtain on the symbolic level

$$
\left[\lambda+\frac{\sigma|\xi|^{2}}{\left(\rho_{1}+\rho_{2}\right) \lambda /|\xi|+4 \eta_{1} \eta_{2} /\left(\eta_{1}+\eta_{2}\right)}\right] h=f_{h} .
$$

Thus the boundary symbol is given by

$$
s(\lambda, \xi):=\lambda+\frac{\sigma|\xi|^{2}}{\left(\rho_{1}+\rho_{2}\right) \lambda /|\xi|+4 \eta_{1} \eta_{2} /\left(\eta_{1}+\eta_{2}\right)} .
$$

4. Mapping properties of $S$. In this section we want to disuss some mapping properties of the operator $S$ associated with the boundary symbol $s(\lambda, \xi)$. For this purpose we introduce some linear operators acting in the base space $X:=L_{p}\left(J \times \mathbb{R}^{n}\right)$ or in ${ }_{0} K_{p}^{s}\left(J ; K_{p}^{r}\left(\mathbb{R}^{n}\right)\right)$, where $1<p<\infty, K \in\{H, W\}, s \geq 0, r \in \mathbb{R}$, and $J=[0, a]$ denotes a finite interval. Note that by a left subscript 0 we indicate that the time traces at $t=0$ of the functions involved vanish whenever this makes sense. For example, if $E$ is a Banach space, then for $K \in\{H, W\}$,

$$
{ }_{0} K_{p}^{s}(J ; E)=\left\{u \in K_{p}^{s}(J, E): u(0)=0\right\}, \quad \text { for } s>1 / p,
$$


and ${ }_{0} K_{p}^{s}(J, E)=K_{p}^{s}(J ; E)$ otherwise. All of the operators defined below will commute in the resolvent sense. As a general reference for the notions and results employed we refer to $[7]$.

We set $G:=\partial_{t}$ in $X$ with domain $\mathcal{D}(G)={ }_{0} H_{p}^{1}\left(J ; L_{p}\left(\mathbb{R}^{n}\right)\right)$. Then it is well-known that $G$ is closed, invertible and sectorial with angle $\pi / 2$, and $-G$ is the generator of a $C_{0}$-semigroup of contractions in $L_{p}\left(\mathbb{R}^{n}\right)$. Moreover, $G$ admits an $H^{\infty}$-calculus in $X$ with $H^{\infty}$-angle $\pi / 2$ as well; see e.g. [11]. The symbol of $G$ is $\lambda$, the time covariable.

Next we set $D_{n}:=-\Delta$, the Laplacian in $L_{p}\left(\mathbb{R}^{n}\right)$ with domain $\mathcal{D}\left(D_{n}\right)=H_{p}^{2}\left(\mathbb{R}^{n}\right)$. It is also well-known that $D_{n}$ is closed and sectorial with angle 0 , and it admits a bounded $H^{\infty}$-calculus which is even $\mathcal{R}$-bounded with $\mathcal{R} H^{\infty}$-angle 0 ; see e.g. [9]. These results also hold for the canonical extension of $D_{n}$ to $X=L_{p}\left(J ; L_{p}\left(\mathbb{R}^{n}\right)\right)$, and also for the fractional power $D_{n}^{1 / 2}$ of $D_{n}$; note that the domain of $D_{n}^{1 / 2}$ is $\mathcal{D}\left(D_{n}^{1 / 2}\right)=L_{p}\left(J ; H_{p}^{1}\left(\mathbb{R}^{n}\right)\right)$. The symbol of $D_{n}$ is $|\xi|^{2}$, that of $D_{n}^{1 / 2}$ is given by $|\xi|$, where $\xi$ means the covariable of $x$.

By the Dore-Venni theorem for sums of commuting sectorial operators, cf. [10, 14], we see that the parabolic operators $L_{j}:=\rho_{j} G+\mu_{j} D_{n}$ with natural domain

$$
\mathcal{D}\left(L_{j}\right)=\mathcal{D}(G) \cap \mathcal{D}\left(D_{n}\right)={ }_{0} H_{p}^{1}\left(J ; L_{p}\left(\mathbb{R}^{n}\right)\right) \cap L_{p}\left(J ; H_{p}^{2}\left(\mathbb{R}^{n}\right)\right)
$$

are closed, invertible and sectorial with angle $\pi / 2$. Moreover, $L_{j}$ also admits a bounded $H^{\infty}$-calculus in $X$ with $H^{\infty}$-angle $\pi / 2$; cf. e.g. [12]. The same results are valid for the operators $F_{j}=L_{j}^{1 / 2}$, their $H^{\infty}$-angle is $\pi / 4$, and their domains are

$$
\mathcal{D}\left(F_{j}\right)=\mathcal{D}\left(G^{1 / 2}\right) \cap \mathcal{D}\left(D_{n}^{1 / 2}\right)={ }_{0} H_{p}^{1 / 2}\left(J ; L_{p}\left(\mathbb{R}^{n}\right)\right) \cap L_{p}\left(J ; H_{p}^{1}\left(\mathbb{R}^{n}\right)\right) .
$$

Note that here we definitely have to use a Bessel potential space. The symbol of $L_{j}$ is $\rho_{j} \lambda+\mu_{j}|\xi|^{2}$ and that of $F_{j}$ is given by $\sqrt{\rho_{j} \lambda+\mu_{j}|\xi|^{2}}$.

We also need the operators $T_{j}$ defined by

$$
T_{1}:=\sqrt{\mu_{1}} F_{1}+\mu_{2} D_{n}^{1 / 2}, \quad T_{2}:=\sqrt{\mu_{2}} F_{2}+\mu_{1} D_{n}^{1 / 2} .
$$

By the Dore-Venni theorem $T_{j}$ are closed, invertible and sectorial with domain $\mathcal{D}\left(T_{j}\right)=$ $\mathcal{D}\left(F_{j}\right) . T_{j}$ admits again a bounded $H^{\infty}$-calculus with $H^{\infty}$-angle $\pi / 4$; cf. [12]. The harmonic mean $T$ of $T_{1}$ and $T_{2}$, i.e.

$$
T:=2 T_{1} T_{2}\left(T_{1}+T_{2}\right)^{-1}=2\left(T_{1}^{-1}+T_{2}^{-1}\right)^{-1}
$$

enjoys the same properties, as another application of the Dore-Venni theorem shows. The symbol of $T$ is given by $\eta:=2 \eta_{1} \eta_{2} /\left(\eta_{1}+\eta_{2}\right)$.

The product $G D_{n}^{-1 / 2}$ with domain

$$
\begin{aligned}
\mathcal{D}\left(G D_{n}^{-1 / 2}\right) & =\left\{h \in \mathcal{R}\left(D_{n}^{1 / 2}\right): D_{n}^{-1 / 2} h \in \mathcal{D}(G)\right\} \\
& ={ }_{0} H_{p}^{1}\left(J ; \dot{H}_{p}^{-1}\left(\mathbb{R}^{n}\right)\right) \cap L_{p}\left(J ; L_{p}\left(\mathbb{R}^{n}\right)\right)
\end{aligned}
$$

is also closed, sectorial and admits a bounded $H^{\infty}$-calculus with $H^{\infty}$-angle $\pi / 2$; cf. [12] Its symbol is given by $\lambda /|\xi|$.

Finally, we consider

$$
N:=\left(\rho_{1}+\rho_{2}\right) G D_{n}^{-1 / 2}+2 T \text {. }
$$


By the Dore-Venni theorem $N$ is closed invertible, and by [12] admits a bounded $H^{\infty}$ calculus as well, with $H^{\infty}$-angle $\pi / 2$. Its domain is given by

$$
\mathcal{D}(N)={ }_{0} H_{p}^{1}\left(J ; \dot{H}_{p}^{-1}\left(\mathbb{R}^{n}\right)\right) \cap L_{p}\left(J ; H_{p}^{1}\left(\mathbb{R}^{n}\right)\right) .
$$

With these definitions, the operator $S$ with symbol $s(\lambda, \xi)$ is represented by

$$
S=G+\sigma D_{n} N^{-1} .
$$

Note that the natural domain of $S$ is given by

$$
\mathcal{D}(S)=\mathcal{D}(G) \cap \mathcal{D}\left(D_{n} N^{-1}\right)={ }_{0} H_{p}^{1}\left(J ; L_{p}\left(\mathbb{R}^{n}\right)\right) \cap L_{p}\left(J ; H_{p}^{1}\left(\mathbb{R}^{n}\right)\right) .
$$

However, since $G$ as well as $D_{n} N^{-1}$ have $H^{\infty}$-angle $\pi / 2$, to obtain invertibility of $S$ the Dore-Venni approach is not directly applicable, unfortunately. This means that we have to study the boundary symbol $s(\lambda,|\xi|)$ directly.

For this purpose note that for complex numbers $w_{j}$ with $\arg w_{j} \in[0, \pi / 2)$, we have $\arg \left(w_{1} w_{2}\right) /\left(w_{1}+w_{2}\right) \in[0, \pi / 2)$ as well. This implies that $s(\lambda,|\xi|)$ has strictly positive real part for each $\lambda$ in the closed right halfplane and for each $\xi \in \mathbb{R}^{n},(\lambda, \xi) \neq(0,0)$, hence $s(\lambda, \mid \xi)$ does not vanish for such $\lambda$ and $\xi$.

We write $s(\lambda,|\xi|)$ in the following way:

$$
s(\lambda, \tau)=\lambda+\sigma \tau k(z), \quad z=\lambda / \tau^{2},
$$

where

$$
k(z)=\left[\left(\rho_{1}+\rho_{2}\right) z+4\left(\frac{1}{\sqrt{\mu_{1}} \sqrt{\rho_{1} z+\mu_{1}}+\mu_{2}}+\frac{1}{\sqrt{\mu_{2}} \sqrt{\rho_{2} z+\mu_{2}}+\mu_{1}}\right)^{-1}\right]^{-1} .
$$

The asymptotics of $k(z)$ are easy to obtain:

$$
k(0)=\frac{1}{2\left(\mu_{1}+\mu_{2}\right)}, \quad z k(z) \rightarrow \frac{1}{\rho_{1}+\rho_{2}} \quad \text { as } z \rightarrow \infty .
$$

This shows that there is a constant $C>0$ such that

$$
|k(z)| \leq \frac{C}{1+|z|}, \quad \operatorname{Re} z \geq 0 .
$$

Hence we see that

$$
|s(\lambda,|\xi|)| \leq C(|\lambda|+|\xi|), \quad \operatorname{Re} \lambda \geq 0, \quad \xi \in \mathbb{R}^{n},
$$

is valid for some constant $C>0$. Next we are going to prove that for each $\varepsilon>0$ there are $\eta>0, c>0$ such that

$$
|s(\lambda, \tau)| \geq c[|\lambda|+|\tau|], \quad \text { for all } \lambda \in \Sigma_{\pi / 2+\eta},|\lambda| \geq \varepsilon, \tau \in \Sigma_{\eta} .
$$

Since $\operatorname{Re} k(z)>0$, by continuity of modulus and argument we therefore obtain an estimate of the form

$$
|s(\lambda, \tau)| \geq c_{0}[|\lambda|+|\tau||k(z)|] \geq c[|\lambda|+|\tau|], \quad \lambda \in \Sigma_{\pi / 2+\eta}, \quad \tau \in \Sigma_{\eta},
$$

provided $|z| \leq M$, with some $\eta>0$ and $c>0$ depending on $M$, but not on $\lambda$ and $\tau$. On 
the other hand, for $|\lambda| \geq \varepsilon|\tau|,|z| \geq M$ we get

$$
\begin{aligned}
|s(\lambda, \tau)| & \geq|\lambda|-\sigma|\tau||k(z)| \\
& \geq \frac{1}{2}[|\lambda|+\varepsilon|\tau|]-\sigma C|\tau| /(1+M) \\
& \geq c[|\lambda|+|\tau|],
\end{aligned}
$$

provided $\varepsilon>2 \sigma C /(1+M)$, and then by extension

$$
|s(\lambda, \tau)| \geq c[|\lambda|+|\tau|], \quad \lambda \in \Sigma_{\pi / 2+\eta}, \quad \tau \in \Sigma_{\eta},|\lambda| \geq \varepsilon|\tau|,|z| \geq M,
$$

provided $\eta>0$ and $c>0$ are sufficiently small, depending on $\lambda_{0}>0$. Combining these two estimates (4.1) follows.

By means of the $\mathcal{R}$-boundedness of the functional calculus for $D_{n}$ in $X$ we see that the operator family $\left(\lambda+D_{n}^{1 / 2}\right) s\left(\lambda, D_{n}^{1 / 2}\right)^{-1}$ is of class $H^{\infty}$ and $\mathcal{R}$-bounded on $\Sigma_{\pi / 2+\eta} \backslash$ $B_{\eta}(0)$. The operator-valued $H^{\infty}$-calculus for $G=\partial_{t}$ on $X$ then implies boundedness of $\left(G+D_{n}^{1 / 2}\right) s\left(G, D_{n}^{1 / 2}\right)^{-1}$ in $X$. This shows that the solution $h$ of the problem $S h=f$ exists, is uniquely determined and belongs to the regularity class

$$
h \in{ }_{0} H_{p}^{1}\left(J ; L_{p}\left(\mathbb{R}^{n}\right)\right) \cap L_{p}\left(J ; H_{p}^{1}\left(\mathbb{R}^{n}\right)\right),
$$

whenever the right hand side is in $X$. Similar results hold for the base spaces ${ }_{0} K_{p}^{s}\left(J ; K_{p}^{r}\left(\mathbb{R}^{n}\right)\right)$, where $s \geq 0, r \in \mathbb{R}, K \in\{H, W\}$. Summarizing, we have

TheOREM 4.1. Let $\rho_{j}, \mu_{j}, \sigma>0,1<p<\infty, s \geq 0, r \in \mathbb{R}$, and $J=[0, a]$. Then the boundary operator $S$ with symbol $s(\lambda, \xi)$,

$$
S:{ }_{0} K_{p}^{s+1}\left(J ; K_{p}^{r}\left(\mathbb{R}^{n}\right)\right) \cap{ }_{0} K_{p}^{s}\left(J ; W K_{p}^{r+1}\left(\mathbb{R}^{n}\right)\right) \rightarrow{ }_{0} K_{p}^{s}\left(J ; K_{p}^{r}\left(\mathbb{R}^{n}\right)\right),
$$

is an isomorphism for $K \in\{H, W\}$.

This result is not valid on the half-line, i.e. for $J=\mathbb{R}_{+}$, since $s(\lambda, \xi)$ is discontinuous at $(0,0)$. However, if one uses spaces with an exponential weight $e^{-\omega t}$ in $t$, then it remains true. In fact, if $h$ is replaced by $e^{-\omega t} h$ and $f_{h}$ by $e^{-\omega t} f_{h}$, then on the symbolic level $s(\lambda, \xi)$ turns into $s(\lambda+\omega, \xi)$, which removes the problem at $(0,0)$. For $\omega>0$, we define the weighted spaces by means of

$$
h \in{ }_{0} K_{p, \omega}^{s}\left(\mathbb{R}_{+} ; K_{p}^{r}\left(\mathbb{R}^{n}\right)\right) \Leftrightarrow e^{-\omega t} h \in{ }_{0} K_{p}^{s}\left(\mathbb{R}_{+} ; K_{p}^{r}\left(\mathbb{R}^{n}\right)\right) .
$$

With this notation we have the following corollary.

Corollary 4.2. Let $\rho_{j}, \mu_{j}, \sigma>0,1<p<\infty, s \geq 0, r \in \mathbb{R}$, and $\omega>0$. Then the boundary operator $S$ with symbol $s(\lambda, \xi)$,

$$
S:{ }_{0} K_{p, \omega}^{s+1}\left(\mathbb{R}_{+} ; K_{p}^{r}\left(\mathbb{R}^{n}\right)\right) \cap{ }_{0} K_{p, \omega}^{s}\left(\mathbb{R}_{+} ; K_{p}^{r+1}\left(\mathbb{R}^{n}\right)\right) \rightarrow{ }_{0} K_{p, \omega}^{s}\left(\mathbb{R}_{+} ; K_{p}^{r}\left(\mathbb{R}^{n}\right)\right),
$$

is an isomorphism for $K \in\{H, W\}$.

In the next sections we present a refined analysis of the boundary symbol to obtain more precise mapping properties for $S$ on $J=\mathbb{R}_{+}$.

5. Singularities and zeros of the boundary symbol. The height function $h$, i.e. the position of the free boundary, is determined by a complicated integro-differential equation 
which is described in terms of its symbol

$$
s(\lambda,|\xi|)=\lambda+\frac{\sigma|\xi|^{2}}{\left(\rho_{1}+\rho_{2}\right) \lambda /|\xi|+4 \eta_{1} \eta_{2} /\left(\eta_{1}+\eta_{2}\right)} .
$$

We now study this symbol in more detail. As before we set $z=\lambda /|\xi|^{2}$, and let $\tau=|\xi|$. This scaling implies

$$
s(\lambda,|\xi|)=m(z, \tau)=\tau^{2}\left(z+\frac{a}{z+\Phi(z)}\right)
$$

where $a=\sigma /\left[\left(\rho_{1}+\rho_{2}\right) \tau\right]$, and

$$
\Phi(z)=\frac{4}{\left(\rho_{1}+\rho_{2}\right)} \frac{\eta_{1}(z) \eta_{2}(z)}{\eta_{1}(z)+\eta_{2}(z)},
$$

with

$$
\eta_{1}(z)=\mu_{2}+\sqrt{\mu_{1}^{2}+\mu_{1} \rho_{1} z}, \quad \eta_{2}(z)=\mu_{1}+\sqrt{\mu_{2}^{2}+\mu_{2} \rho_{2} z} .
$$

So in particular, if $\rho_{1}=\rho_{2}=\rho$ and $\mu_{1}=\mu_{2}=\mu$, i.e. if the phases are hydrodynamically indistinguishable, then

$$
\Phi(z)=\frac{\mu}{\rho}(1+\sqrt{1+\rho z / \mu}),
$$

and if $\rho_{2}=\mu_{2}=0, \rho_{1}=\rho, \mu_{1}=\mu$, i.e. in the one-phase case, we have

$$
\Phi(z)=\frac{4 \mu}{\rho} \frac{\sqrt{1+\rho z / \mu}}{1+\sqrt{1+\rho z / \mu}},
$$

which converges to $4 \mu / \rho$ as $z \rightarrow \infty$. Note the difference in the asymptotic behaviour as $z \rightarrow \infty$ of the two-phase and the one-phase case!

Observe that $\eta_{j}(z)$ is holomorphic in the sliced plane $\mathbb{C} \backslash\left(-\infty,-\mu_{j} / \rho_{j}\right]$, it has a branch cut at $-\rho_{j} / \mu_{j}$. If $\arg z \in[0, \pi]$ then $\arg \eta_{j}(z) \in[0, \pi / 2)$, and $\eta_{j}(z) \neq 0$. Therefore the harmonic mean of $\eta_{j}(z)$, i.e. $\Phi(z)$, is holomorphic in $\mathbb{C} \backslash\left(-\infty, z_{0}\right]$, where $z_{0}:=$ $-\min \left\{\mu_{1} / \rho_{1}, \mu_{2} / \rho_{2}\right\}<0$. If $\arg z \in[0, \pi]$ then $\arg \Phi(z) \in[0, \pi / 2), \Phi(z) \neq 0$, and $\Phi(0)=$ : $\Phi_{0}=2\left(\mu_{1}+\mu_{2}\right) /\left(\rho_{1}+\rho_{2}\right)$. Moreover, note that as $z \rightarrow \infty$ we have the asymptotics $\eta_{j}(z) \sim \sqrt{\mu_{j} \rho_{j} z}$, hence

$$
\Phi(z) \sim \Phi_{\infty} \sqrt{z}, \text { as } z \rightarrow \infty, \text { where } \Phi_{\infty}=\frac{4}{\rho_{1}+\rho_{2}} \frac{\sqrt{\mu_{1} \rho_{1}} \sqrt{\mu_{2} \rho_{2}}}{\sqrt{\mu_{1} \rho_{1}}+\sqrt{\mu_{2} \rho_{2}}} .
$$

This mapping behaviour of $\Phi$ shows that the function $z+\Phi(z)$ does not admit zeros in $\mathbb{C} \backslash(-\infty, 0)$.

Assume first that $z_{0}+\Phi\left(z_{0}\right)<0$. The function $\Phi$ is strictly increasing on the real interval $\left(z_{0}, \infty\right)$ and $\Phi(0)=\Phi_{0}>0$. Therefore, $z+\Phi(z)$ has exactly one real zero $z_{\infty} \in\left(z_{0}, 0\right)$ which is of order one. At this point $m(z, \tau)$ has a pole of first order, for all $\tau, a \neq 0$. Consequently, for fixed $\tau \neq 0, \infty$, the function $m^{-1}(z, \tau)$ is meromorphic w.r.t. $z \in \mathbb{C} \backslash\left(-\infty, z_{0}\right]$. We are next looking for the zeros of $m(z, \tau)$ in dependence of $\tau$, which means that we have to look at the zeros of the function

$$
\Psi_{a}(z)=z(z+\Phi(z))+a, \quad z \notin\left(-\infty, z_{0}\right] .
$$


For this purpose we first consider $a=0$. Obviously, $\Psi_{0}(z)$ has precisely the two zeros $z_{1}(0)=0$ and $z_{2}(0)=z_{\infty}$. With

$$
\Psi_{a}^{\prime}(z)=2 z+\Phi(z)+z \Phi^{\prime}(z), \quad \Phi^{\prime}(z)=\frac{\rho_{1}+\rho_{2}}{4}\left[\eta_{1}^{\prime}(z) \eta_{1}^{-2}(z)+\eta_{2}^{\prime}(z) \eta_{2}^{-2}(z)\right] \Phi^{2}(z),
$$

we obtain $\Psi_{0}^{\prime}(0)=\Phi_{0}>0$, as well as $\Psi_{0}^{\prime}\left(z_{\infty}\right)=z_{\infty}\left(1+\Phi^{\prime}\left(z_{\infty}\right)\right)<0$. Hence the implicit function theorem yields analytic functions $z_{j}(a)$ in a neighborhood of $a=0$ such that $z_{1}(0)=0, z_{2}(0)=z_{\infty}$ and $z_{j}(a)$ are the only zeros of $\Psi_{a}(z)$ for a close to zero. Differentiating the equations $\Psi_{a}\left(z_{j}(a)\right)=0$ we obtain the expressions

$$
z_{j}^{\prime}(0)=-1 / \Psi_{0}^{\prime}\left(z_{j}(0)\right), \quad z_{j}^{\prime \prime}(0)=-\Psi_{0}^{\prime \prime}\left(z_{j}(0)\right) /\left[\Psi_{0}^{\prime}\left(z_{j}(0)\right)\right]^{3} .
$$

This yields

$$
z_{1}^{\prime}(0)=-1 / \Phi_{0}<0, \quad z_{2}^{\prime}(0)=-1 /\left[z_{\infty}\left(1+\Phi^{\prime}\left(z_{\infty}\right)\right)\right]>0,
$$

and with $\Psi_{0}^{\prime \prime}(0)=3$, the asymptotics

$$
z_{1}(a)=-a / \Phi_{0}-3 a^{2} / 2 \Phi_{0}^{3}+O\left(a^{3}\right), \quad \text { as } a \rightarrow 0,
$$

and

$$
\left.z_{2}(a)=z_{\infty}-a /\left[z_{\infty}\left(1+\Phi^{\prime}\left(z_{\infty}\right)\right)\right]-a^{2} \Psi_{0}^{\prime \prime}\left(z_{\infty}\right) / 2\left[\Psi_{0}^{\prime}\left(z_{\infty}\right)\right)\right]^{3}+O\left(a^{3}\right), \quad \text { as } a \rightarrow 0 .
$$

For the corresponding zeros of $s(\lambda,|\xi|)$ this means to first order

$$
\begin{gathered}
\lambda_{1}(|\xi|)=-|\xi| \sigma /\left[2\left(\mu_{1}+\mu_{2}\right)\right]+O(1), \\
\lambda_{2}(|\xi|)=z_{\infty}|\xi|^{2}-|\xi| \sigma /\left[\left(\rho_{1}+\rho_{2}\right) z_{\infty}\left(1+\Phi^{\prime}\left(z_{\infty}\right)\right)\right]+O(1),
\end{gathered}
$$

as $|\xi| \rightarrow \infty$. Thus for large $|\xi|$ there are exactly two negative real zeros of $s(\lambda,|\xi|)$ which tend to $-\infty$ as $|\xi| \rightarrow \infty$.

Next consider the case $\xi=0$, i.e. $a \rightarrow \infty$. Then for the zeros $z_{j}(a)$ we necessarily have $\left|z_{j}(a)\right| \rightarrow \infty$, hence the term $z \Phi(z)$ in the function $\Psi_{a}(z)$ is of lower order, compared to $z^{2}$. Thus the lowest order asymptotics of the zeros of $\Psi_{a}(z)$ are given by the equation $z^{2}+a=0$, which implies

$$
z_{j}(a) \sim \pm i \sqrt{a}, \quad \text { as } a \rightarrow \infty
$$

For the zeros of $s(\lambda,|\xi|)$ this yields the asymptotics

$$
\lambda_{j}(|\xi|) \sim \pm i|\xi|^{3 / 2} \sqrt{\sigma /\left(\rho_{1}+\rho_{2}\right)}, \quad \text { as } \xi \rightarrow 0 .
$$

A more precise asymptotics is obtained as follows. Consider the equation $\Psi_{a}(z)=z^{2}+$ $z \Phi(z)+a=0$ as a quadratic equation for $z$. Then

$$
\begin{aligned}
z_{j}(a) & =-\Phi\left(z_{j}(a)\right) / 2 \pm i \sqrt{a-\Phi^{2}\left(z_{j}(a)\right) / 4} \\
& = \pm i \sqrt{a} \sqrt{1-\Phi^{2}\left(z_{j}(a)\right) / 4 a}-\Phi\left(z_{j}(a)\right) / 2 \\
& \sim \pm i \sqrt{a} \sqrt{1-\Phi_{\infty}^{2} z_{j}(a) / 4 a}-\Phi_{\infty} \sqrt{z_{j}(a)} / 2 \\
& \sim \pm i \sqrt{a}\left(1-\Phi_{\infty}^{2}( \pm i \sqrt{a} / 8 a)\right)-\Phi_{\infty} \sqrt{ \pm i} a^{1 / 4} / 2 \\
& = \pm i \sqrt{a}-\frac{\Phi_{\infty}}{2 \sqrt{2}} a^{1 / 4}(1 \pm i)+\frac{\Phi_{\infty}^{2}}{8}
\end{aligned}
$$


as $a \rightarrow \infty$. This implies

$$
\lambda_{j}(|\xi|) \sim \pm i|\xi|^{3 / 2}\left(\frac{\sigma}{\rho_{1}+\rho_{2}}\right)^{1 / 2}-|\xi|^{7 / 4} \frac{\Phi_{\infty}}{2 \sqrt{2}}(1 \pm i)\left(\frac{\sigma}{\rho_{1}+\rho_{2}}\right)^{1 / 4}+|\xi|^{2} \frac{\Phi_{\infty}^{2}}{8},
$$

as $\xi \rightarrow 0$. By means of the scaling $z=\sqrt{a} w$ and the implicit function theorem, it is not difficult to see that $\lambda_{j}$ is an analytic function of $\tau^{1 / 4}$ in a neighbourhood of $\tau=0$.

Since for finite $a$ away from zero there are no zeros of $\Psi_{a}(z)$ in the closed right half-plane, and since they cannot escape to infinity nor touch the branch cut, there are two continuous curves of zeros $z_{j}(a), j=1,2$, of $\Psi_{a}(z)$. These are negative real for small positive $a$ and complex conjugate for large $a$. Therefore there must be a real double zero of $\Psi_{a}(z)$, for some value $a_{*} \in(0, \infty)$. To determine $a_{*}$ and $z_{*}:=z_{j}\left(a_{*}\right)$ we have to consider the two equations

$$
\Psi_{a}(z)=z(z+\Phi(z))+a=0, \quad \Psi_{a}^{\prime}(z)=2 z+\Phi(z)+z \Phi^{\prime}(z)=0 .
$$

The first one determines $a_{*}=-z_{*}\left(z_{*}+\Phi\left(z_{*}\right)\right)>0$ in terms of $z_{*}$, and the second one $z_{*}$ since it no longer depends on $a$. Since $\Phi(z)$ is increasing and concave in $\left[z_{0}, 0\right]$, the function $\Psi_{0}(z)$ is strictly convex in $\left(z_{0}, 0\right]$, hence has a unique minimum $z_{*} \in\left(z_{0}, 0\right)$; actually $z_{*}>z_{\infty}$ since $\Psi_{0}^{\prime}\left(z_{\infty}\right)<0$. Therefore, the curves of zeros $z_{j}(a)$ are analytic in $[0, \infty) \backslash\left\{a_{*}\right\}$, negative real for $a<a_{*}$, complex conjugate for $a>a_{*}$, and double at $a=a_{*}$ with value $z_{j}\left(a_{*}\right)=z_{*}$. Note that $a_{*}$ and $z_{*}$ are determined by the parameters $\mu_{j}$ and $\rho_{j}$ only, just the corresponding value of $\tau_{*}$ depends also on the coefficient of surface tension $\sigma>0$.

On the other hand, if $z_{0}+\Phi\left(z_{0}\right) \geq 0$, then $z+\Phi(z)$ has no zeros in $\left(z_{0}, 0\right)$. We then define $a_{0}=-\Psi_{0}\left(z_{0}\right)$. Since $\Psi_{0}^{\prime}\left(z_{0}\right)=-\infty$, we find a continuous function $z_{2}:\left[a_{0}, a_{0}+\delta\right] \rightarrow$ $\left[z_{0}, 0\right)$ analytic in $\left(a_{0}, a_{0}+\delta\right)$ such that $z_{2}\left(a_{0}\right)=z_{0}$ and $\Psi_{a}\left(z_{2}(a)\right) \equiv 0$. In case $a_{0}=0$, i.e. $z_{0}+\Phi\left(z_{0}\right)=0$ we have the asymptotics

$$
z_{2}(a) \sim z_{0}+O\left(a^{2}\right), \quad a \sim 0 .
$$

Let us summarize what we have shown above.

Proposition 5.1. Suppose $\rho_{j}, \mu_{j}, \sigma>0$, let $z_{0}:=-\min \left\{\mu_{1} / \rho_{1}, \mu_{2} / \rho_{2}\right\}$, and let $z_{*}$ denote the unique zero of $\Psi_{0}^{\prime}(z)$ in $\left(z_{0}, 0\right)$. Set $a_{*}=-\Psi_{0}\left(z_{*}\right)$, let $a_{0}:=-\Psi_{0}\left(z_{0}\right)$, and $a=\sigma /\left[\left(\rho_{1}+\rho_{2}\right) \tau\right], \tau>0$. Then

(i) $m(z, \tau)$ is holomorphic w.r.t. $z \in \mathbb{C} \backslash\left(-\infty, z_{0}\right]$, except for one simple pole in case $a_{0}<0$, and $z_{1,2}\left(a_{*}\right)=z_{*}$ is a double zero of $m\left(z, \tau_{*}\right)$.

(ii) For $a_{*}<$ a there are precisely two zeros of $m(z, \tau)$, with asymptotics

$$
z_{1,2}(a) \sim \pm i \sqrt{a}-\frac{\Phi_{\infty}}{2 \sqrt{2}} a^{1 / 4}(1 \pm i)+\frac{\Phi_{\infty}^{2}}{8}, \quad a \rightarrow \infty .
$$

(iii) If $a_{0}<0$ and $0<a<a_{*}$, then $m(z, \tau)$ has exactly two real zeros, with asymptotics

$$
\begin{aligned}
& z_{1}(a)=-a / \Phi_{0}+O\left(a^{2}\right), \quad a \rightarrow 0, \\
& z_{2}(a)=z_{\infty}-a /\left[z_{\infty}\left(1+\Phi^{\prime}\left(z_{\infty}\right)\right)\right]+O\left(a^{2}\right), \quad a \rightarrow 0,
\end{aligned}
$$

where $z_{\infty} \in\left(z_{0}, 0\right)$ denotes the unique zero of $z+\Phi(z)$. 
(iv) If $a_{0}=0$ then $z_{\infty}=z_{0}$, and for $0 \leq z<a_{*}$ there are precisely two real zeros, with asymptotics

$$
z_{1}(a)=-a / \Phi_{0}+O\left(a^{2}\right), \quad z_{2}(a)=z_{0}+O\left(a^{2}\right), \quad a \rightarrow 0
$$

(v) If $a_{0}>0$ there are precisely two zeros for $a_{0} \leq z<a_{*}$, and only one zero $z_{1}(a)$ for $0 \leq a<a_{0}$, with asymptotics

$$
z_{1}(a)=-a / \Phi(0)+O\left(a^{2}\right), \quad a \rightarrow 0
$$

Let us point out an essential difference in the asymptotics of the zeros of $s(\lambda,|\xi|)$ for small frequencies between the one- and two-phase case. In fact, since in the one-phase case we have $\Phi(z) \rightarrow 4 \eta / \rho$ as $z \rightarrow \infty$, we obtain for small frequencies $\tau$, i.e. large $a$

$$
\begin{aligned}
z_{j}(a) & =-\Phi\left(z_{j}(a)\right) / 2 \pm i \sqrt{a-\Phi^{2}\left(z_{j}(a)\right) / 4} \\
& = \pm i \sqrt{a} \sqrt{1-\Phi^{2}\left(z_{j}(a)\right) / 4 a}-\Phi\left(z_{j}(a)\right) / 2 \\
& \sim \pm i \sqrt{a} \sqrt{1-(4 \mu / \rho)^{2} / 4 a}-(4 \mu / \rho) / 2 \\
& \sim \pm i \sqrt{a}\left(1-2 \mu^{2} /\left(\rho^{2} a\right)\right)-2 \mu / \rho \\
& = \pm i \sqrt{a} \mp 2 i \mu^{2} /\left(\rho^{2} \sqrt{a}\right)-2 \mu / \rho
\end{aligned}
$$

as $a \rightarrow \infty$. This implies

$$
\lambda_{j}(|\xi|) \sim \pm i|\xi|^{3 / 2} \sqrt{\sigma / \rho} \mp 2 i(\mu / \rho)^{2} \sqrt{\rho / \sigma}|\xi|^{5 / 2}-2 \mu|\xi|^{2} / \rho,
$$

as $\xi \rightarrow 0$. By means of the scaling $z=\sqrt{a} w$ and the implicit function theorem, it is not difficult to see that $\lambda_{j}$ is an analytic function of $\tau^{1 / 2}$ in a neighbourhood of $\tau=0$, rather than of $\tau^{1 / 4}$ as in the two-phase case. We will see consequences of this fact in section 7 . On the other hand, for large frequencies the asymptotics of the one- and two-phase cases coincide qualitatively.

The interpretation of the zeros of $s(\lambda,|\xi|)$ is as follows. The function

$$
h(t, x):=c e^{\lambda t+i \xi \cdot x}, \quad t \in \mathbb{R}, \quad x \in \mathbb{R}^{n},
$$

is a solution of the homogeneous boundary problem if and only if $s(\lambda,|\xi|)=0$. Our analysis shows that such a function $h$ is a solution if and only if $\lambda=\lambda_{j}(|\xi|), j=1,2$. For large $|\xi|$ this yields solutions which do not oscillate in time and converge to zero as $t \rightarrow \infty$ exponentially, with rate either $|\xi|^{2}\left|z_{\infty}\right|$ or $|\xi| \sigma /\left[2\left(\mu_{1}+\mu_{2}\right)\right]$. On the other hand, if $|\xi|$ is small, then we have waves oscillating in time with frequency $|\xi|^{3 / 2} \sqrt{\sigma /\left(\rho_{1}+\rho_{2}\right)}$ which are exponentially damped with attenuation

$$
|\xi|^{7 / 4}\left(\Phi_{\infty} / 2 \sqrt{2}\right)\left[\sigma /\left(\rho_{1}+\rho_{2}\right)\right]^{1 / 4} .
$$

Note that the attenuation behaves like $|\xi|^{7 / 4}$ (like $|\xi|^{2}$ in the one-phase case), the time frequency like $|\xi|^{3 / 2}$ and the wave length in space like $1 /|\xi|$, and the wave speed like $|\xi|^{1 / 2}$. For small $\xi$ the attenuation is small compared to the frequency. These waves show to some extent a hyperbolic character of the boundary problem. For large $|\xi|$ the boundary problem shows parabolic behavior, but for small $|\xi|$ it exhibits hyperbolic properties. 
6. High frequency analysis. To continue, we next compute the residues of $m^{-1}(z, \tau)$ at the poles $z_{j}(a)$. Since for $a \neq a_{*}$ these poles are simple, we obtain

$$
c_{j}(\tau):=\operatorname{Res} m^{-1}(z, \tau)_{\left.\right|_{z=z_{j}}}=\lim _{z \rightarrow z_{j}}\left(z-z_{j}\right) m^{-1}(z, \tau)=\frac{\tau^{-2}}{1-\frac{z_{j}^{2}}{a}\left(1+\Phi^{\prime}\left(z_{j}\right)\right)} .
$$

For $a \rightarrow \infty$ we have $\left|z_{j}(a)\right| \rightarrow \infty$, hence with $\Phi^{\prime}(z) \rightarrow 0$ as $|z| \rightarrow \infty$, this implies

$$
c_{j}(\tau) \sim \tau^{-2} \frac{1}{2+\Phi^{\prime}\left(z_{j}\right)} \sim \frac{\tau^{-2}}{2} .
$$

On the other hand, for $a \rightarrow 0$ we obtain

$$
c_{1}(\tau) \sim \tau^{-2}\left(1+\frac{3 a}{2 \Phi_{0}^{2}}\right)
$$

and in case $a_{0}<0$

$$
c_{2}(\tau) \sim-\frac{\tau^{-2} a}{z_{\infty}^{2}\left(1+\Phi^{\prime}\left(z_{\infty}\right)\right)} .
$$

With $m_{3}(z, \tau)=m_{1}(z, \tau)+m_{2}(z, \tau), m_{j}(z, \tau)=c_{j}(\tau) /\left(z-z_{j}(a)\right)$, we may decompose the symbol $m^{-1}(z, \tau)$ as

$$
m^{-1}(z, \tau)=m_{0}(z, \tau)+m_{3}(z, \tau), \quad m_{3}(z, \tau)=\frac{\alpha(\tau) z+\beta(\tau)}{z^{2}+\gamma(\tau) z+\delta(\tau)},
$$

where $\alpha(\tau)=c_{1}(\tau)+c_{2}(\tau), \beta(\tau)=-\left(c_{1}(\tau) z_{2}(a)+c_{2}(\tau) z_{1}(a)\right), \delta(\tau)=z_{1}(a) z_{2}(a)$ and $\gamma(\tau)=-\left(z_{1}(a)+z_{2}(a)\right)$. Note that the coefficients $\alpha, \beta, \gamma, \delta$ are real for all values of $\tau>0$, and $\gamma, \delta$ are positive, and moreover they are analytic w.r.t. $\tau \in \Sigma_{\varepsilon}$, for some small $\varepsilon>0$.

By Cauchy's theorem we get the following representation for $m_{0}$, contracting the contour to the branch cut of $m$ :

$$
m_{0}(z, \tau)=-\frac{a}{2 \pi i \tau^{2}} \int_{\left|z_{0}\right|}^{\infty} \frac{\left(\Phi_{+}(r)-\Phi_{-}(r)\right) d r /(z+r)}{\left(r^{2}+a\right)^{2}-\left(r^{2}+a\right) r\left(\Phi_{+}(r)+\Phi_{-}(r)\right)+r^{2} \Phi_{+}(r) \Phi_{-}(r)} .
$$

Here the functions $\Phi_{ \pm}(r)$ are defined as the upper and lower limits of $\Phi$ on the branch cut, i.e.

$$
\Phi_{ \pm}(r)=\lim _{\theta \rightarrow 0+} \Phi\left(r e^{ \pm i(\pi-\theta)}\right), \quad r>r_{0}:=\left|z_{0}\right| .
$$

Since $\Phi_{-}(r)=\overline{\Phi_{+}(r)}$, this yields

$$
m_{0}(z, \tau)=-\frac{a}{\pi \tau^{2}} \int_{r_{0}}^{\infty} \frac{\operatorname{Im} \Phi_{+}(r) d r /(z+r)}{\left(r^{2}+a-r \operatorname{Re} \Phi_{+}(r)\right)^{2}+r^{2}\left|\operatorname{Im} \Phi_{+}(r)\right|^{2}} .
$$

As $r \rightarrow \infty$ we have $\Phi(r) \sim \Phi_{\infty} \sqrt{r}$, hence $c \sqrt{r} \leq\left|\Phi_{ \pm}(r)\right| \leq c^{-1} \sqrt{r}$, for $r \in\left[r_{0}, \infty\right)$, with some constant $c>0$. This implies the estimate

$$
\left|m_{0}(z, \tau)\right| \leq \frac{C}{\tau^{3}(|z|+1)}, \quad z \in \Sigma_{\pi / 2+\varepsilon}, \quad \tau \in \Sigma_{\varepsilon}, \quad|\tau| \geq R .
$$

Therefore, the symbol $m_{0}(z, \tau)$ multiplied by a cut-off function $\chi(|\xi|)$ which is zero in $B_{R}(0)$ corresponds to a bounded linear operator which maps $L_{p}\left(\mathbb{R}_{+} ; L_{p}\left(\mathbb{R}^{n}\right)\right)$ into ${ }_{0} H_{p}^{1}\left(\mathbb{R}_{+} ; H_{p}^{1}\left(\mathbb{R}^{n}\right)\right) \cap L_{p}\left(\mathbb{R}_{+} ; H_{p}^{3}\left(\mathbb{R}^{n}\right)\right)$. More precisely, let

$$
g(r, \tau)=\frac{-\sigma \operatorname{Im} \Phi_{+}(r)}{\left.\pi\left(\rho_{1}+\rho_{2}\right)\left[r\left(r-\Phi_{+}(r)\right)+\sigma /\left(\rho_{1}+\rho_{2}\right) \tau\right)^{2}+r^{2}\left(\operatorname{Im} \Phi_{+}(r)\right)^{2}\right]} .
$$


Then by the Dunford calculus for the bounded linear operator $D_{n}^{-1 / 2}$, the operator function $g\left(r, D_{n}^{-1 / 2}\right)$ is well-defined and bounded by $c / r^{4}$. A Dore-Venni argument implies that $G+r D_{n}$ with natural domain is invertible and $G\left(G+r D_{n}\right)^{-1}$ as well as $r D_{n}\left(G+r D_{n}\right)^{-1}$ are bounded, uniformly w.r.t. $r>0$. This implies the representation

$$
M_{0}=D_{n}^{-1 / 2} \int_{r_{0}}^{\infty} g\left(r, D_{n}^{1 / 2}\right)\left(G+r D_{n}\right)^{-1} d r
$$

which is absolutely convergent in the operator norm.

Let us next look at the symbol

$$
m_{2}(z, \tau)=\frac{c_{2}(\tau)}{z-z_{2}(a)},
$$

for large $\tau$, in case $a_{0}<0$. From the asymptotics of $c_{j}$ and $z_{j}$ we have

$$
m_{2}(z, \tau)=\frac{c_{2}(|\xi|)|\xi|^{2}}{\lambda-\lambda_{2}(a)} \sim \frac{-c_{\infty}|\xi|^{-1}}{\lambda+\left|z_{\infty}\right||\xi|^{2}}
$$

where $c_{\infty}=\sigma /\left[\left(\rho_{1}+\rho_{2}\right) z_{\infty}^{2}\left(1+\Phi^{\prime}\left(z_{\infty}\right)\right)\right]>0$ is a constant only depending on the parameters. These asymptotics show that the symbol $m_{2}(z, \tau)$ multiplied by $\chi(|\xi|)$ corresponds to an operator which is bounded from $L_{p}\left(\mathbb{R}_{+} ; L_{p}\left(\mathbb{R}^{n}\right)\right)$ to ${ }_{0} H_{p}^{1}\left(\mathbb{R}_{+} ; H_{p}^{1}\left(\mathbb{R}^{n}\right)\right) \cap$ $L_{p}\left(\mathbb{R}_{+} ; H_{p}^{3}\left(\mathbb{R}^{n}\right)\right)$ as well. More precisely, since $c_{2}(\tau)$ as well as $z_{2}(a)$ are holomorphic w.r.t. in a neighborhood of $a=0$, the Dunford calculus yields with the asymptotics for $c_{2}$ and $z_{2}$ the representation

$$
\left(M_{2} f\right)(t)=\int_{0}^{t}\left(c_{2}^{0}+P_{2}\left(D_{n}^{1 / 2}\right)\right) e^{-\left(\left|z_{\infty}\right| D_{n}+c_{2}^{1} D_{n}^{1 / 2}+c_{2}^{2}+Q_{2}\left(D_{n}^{1 / 2}\right)\right)(t-s)} f(s) d s, \quad t>0 .
$$

Here $P_{2}\left(D_{n}^{1 / 2}\right)$ and $Q_{2}\left(D_{n}^{1 / 2}\right)$ are bounded linear operators which converge to 0 as $R \rightarrow \infty$. The constants $c_{2}^{j}, j=0,1,2$, are positive. In particular, the kernel has exponential decay in $t$, and behaves like the diffusion semigroup. We set $M_{2}=0$ in case $a_{0} \geq 0$.

On the other hand, for

$$
m_{1}(z, \tau)=\frac{c_{1}(\tau)}{z-z_{1}(a)}
$$

we obtain from the asymptotics

$$
m_{1}(z, \tau)=\frac{c_{1}(|\xi|)|\xi|^{2}}{\lambda-\lambda_{1}(a)} \sim \frac{1}{\lambda+|\xi| \sigma /\left[2\left(\mu_{1}+\mu_{2}\right)\right]} .
$$

This is the principal part of the symbol $s^{-1}(\lambda,|\xi|)$ for large $|\xi|$. It corresponds to a linear operator which is bounded from $L_{p}\left(\mathbb{R}_{+} ; L_{p}\left(\mathbb{R}^{n}\right)\right)$ into ${ }_{0} H_{p}^{1}\left(\mathbb{R}_{+} ; L_{p}\left(\mathbb{R}^{n}\right)\right) \cap L_{p}\left(\mathbb{R}_{+} ; H_{p}^{1}\left(\mathbb{R}^{n}\right)\right)$, only. By the Dunford calculus, the operator $M_{1}$ is given by

$$
\left(M_{1} f\right)(t)=\int_{0}^{t}\left(I+P_{1}\left(D_{n}^{1 / 2}\right)\right) e^{-\left(D_{n}^{1 / 2}+c_{1}^{1}+Q_{1}\left(D_{n}^{1 / 2}\right)\right)(t-s)} f(s) d s, \quad t>0,
$$

where $P\left(D_{n}\right)$ and $Q\left(D_{n}\right)$ are bounded with $L_{p}$-norms tending to zero as $R \rightarrow \infty$, and $c_{1}^{1}$ is a positive constant. Note that the kernel of $M_{1}$ is exponentially decaying and behaves like the Poisson semigroup, in contrast to $M_{2}$.

Summarizing we have proved the following result. 
Theorem 6.1. Let $\rho_{j}, \mu_{j}, \sigma>0, j=1,2,1<p<\infty$. Fix any $R>0$ large enough, and let

$$
\mathcal{S}_{R^{c}}^{\prime}\left(\mathbb{R}^{n}\right):=\left\{g \in \mathcal{S}^{\prime}\left(\mathbb{R}^{n}\right): \operatorname{supp}(\mathcal{F} g) \subset\left(B_{R}(0)\right)^{c}\right\} .
$$

Then $S^{-1}$ decomposes as $S^{-1}=M_{0}+M_{1}+M_{2}$, such that

$$
M_{0}, M_{2}: L_{p}\left(\mathbb{R}_{+} ; L_{p}\left(\mathbb{R}^{n}\right) \cap \mathcal{S}_{R^{c}}^{\prime}\left(\mathbb{R}^{n}\right)\right) \rightarrow{ }_{0} H_{p}^{1}\left(\mathbb{R}_{+} ; H_{p}^{1}\left(\mathbb{R}^{n}\right)\right) \cap L_{p}\left(\mathbb{R}_{+} ; H_{p}^{3}\left(\mathbb{R}^{n}\right)\right)
$$

and

$$
M_{1}: L_{p}\left(\mathbb{R}_{+} ; L_{p}\left(\mathbb{R}^{n}\right) \cap \mathcal{S}_{R^{c}}^{\prime}\left(\mathbb{R}^{n}\right)\right) \rightarrow{ }_{0} H_{p}^{1}\left(\mathbb{R}_{+} ; L_{p}\left(\mathbb{R}^{n}\right)\right) \cap L_{p}\left(\mathbb{R}_{+} ; H_{p}^{1}\left(\mathbb{R}^{n}\right)\right) .
$$

are bounded.

7. Low frequency analysis. Thus it remains to study the symbol $s^{-1}(\lambda,|\xi|)$ for small values of $|\xi|$. Note that in this situation $D_{n}^{1 / 2}$ is bounded but not invertible. To begin, we first consider $m_{0}(z, \tau)$. Set $r_{0}=\left|z_{0}\right|$. Then an upper bound for large $a$ is easily seen to be

$$
\left|m_{0}(z, \tau)\right| \leq C a^{3} \int_{r_{0}}^{\infty} \frac{\sqrt{r} d r}{\left(r^{2}+a\right)^{2}(|z|+r)} .
$$

By positivity of the integrand involved, the latter integral also yields a lower bound, for $z>0$ at least. We scale this integral in the following way:

$$
a^{3} \int_{r_{0}}^{\infty} \frac{\sqrt{r} d r}{\left(r^{2}+a\right)^{2}(|z|+r)}=a^{5 / 4} \int_{r_{0} / \sqrt{a}}^{\infty} \frac{\sqrt{s} d s}{\left(s^{2}+1\right)^{2}(s+|z| / \sqrt{a})} .
$$

Estimating the term $1 /(s+|z| / \sqrt{a})$ by either $1 / s$ or $\sqrt{a} /|z|$ we obtain

$$
\left|m_{0}(z, \tau)\right| \leq C a^{5 / 4} \min \{1, \sqrt{a} /|z|\} \leq C a^{5 / 4} \frac{\sqrt{a}}{|z|+\sqrt{a}}=C \frac{a^{7 / 4}}{|z|+\sqrt{a}} \leq C \frac{|\xi|^{1 / 4}}{|\lambda|+|\xi|^{3 / 2}} .
$$

Up to constants, for small $|\xi|$, this estimate is optimal, and it implies that the symbol $m_{0}(z,|\xi|)[1-\chi(|\xi|)]$ induces a bounded linear operator $M_{0}$ from $L_{p}\left(\mathbb{R}_{+} ; \dot{H}_{p}^{-5 / 4}\left(\mathbb{R}^{n}\right)\right)$ into ${ }_{0} H_{p}^{1}\left(\mathbb{R}_{+} ; H_{p}^{m}\left(\mathbb{R}^{n}\right)\right)$ for all $m \in \mathbb{N}_{0}$. Moreover, the time derivative $\partial_{t} M_{0}$ of $M_{0}$ even maps $L_{p}\left(\mathbb{R}_{+} ; L_{p}\left(\mathbb{R}^{n}\right)\right)$ into $L_{p}\left(\mathbb{R}_{+} ; H_{p}^{m}\left(\mathbb{R}^{n}\right) \cap \dot{H}_{p}^{-1 / 4}\left(\mathbb{R}^{n}\right)\right)$ for all $m \in \mathbb{N}_{0}$.

The lowest order asymptotics of $c_{j}$ and $z_{j}$ yield in the case of small $|\xi|$

$$
m_{3}(z, \tau) \sim \frac{1}{2}\left[\frac{1}{\lambda+i c_{0}|\xi|^{3 / 2}}+\frac{1}{\lambda-i c_{0}|\xi|^{3 / 2}}\right]=\frac{\lambda}{\lambda^{2}+c_{0}^{2}|\xi|^{3}},
$$

where $c_{0}=\left[\sigma /\left(\rho_{1}+\rho_{2}\right)\right]^{1 / 2}$. This is the symbol corresponding to the cosine family $C(t)=$ $\cos \left(c_{0} D^{3 / 4} t\right)$ generated by the operator $c_{0}^{2} D_{n}^{3 / 2}$.

Taking the first order asymptotics instead we obtain

$$
m_{1}(z, \tau) \sim \frac{1}{2} \frac{1}{\lambda+\tilde{c}_{0}|\xi|^{7 / 4}-i c_{0}|\xi|^{3 / 2}} .
$$

This implies that the symbol $m_{3}(z, \tau)(1-\chi(|\xi|))$ gives rise to a bounded linear operator which maps from the space $L_{p}\left(\mathbb{R}_{+} ; \dot{H}_{p}^{-7 / 4}\left(\mathbb{R}^{n}\right)\right)$ into $L_{p}\left(\mathbb{R}_{+} ; H_{p}^{m}\left(\mathbb{R}^{n}\right)\right)$, and its time derivative maps $L_{p}\left(\mathbb{R}_{+} ; \dot{H}_{p}^{-1 / 4}\left(\mathbb{R}^{n}\right)\right)$ into $L_{p}\left(\mathbb{R}_{+} ; H_{p}^{m}\left(\mathbb{R}^{n}\right)\right)$, for all $m \in \mathbb{N}_{0}$. This shows that $m_{3}$ is really the principal part of $m$ for small values of $|\xi|$. 
Theorem 7.1 (Two-phase case). Let $\rho_{j}, \mu_{j}, \sigma>0, j=1,2,1<p<\infty$. Fix any $R>0$ small enough, and let

$$
\mathcal{S}_{R}^{\prime}\left(\mathbb{R}^{n}\right):=\left\{g \in \mathcal{S}^{\prime}\left(\mathbb{R}^{n}\right): \operatorname{supp}(\mathcal{F} g) \subset \bar{B}_{R}(0)\right\} .
$$

Then $S^{-1}$ decomposes as $S^{-1}=M_{0}+M_{1}$, such that

$$
\begin{aligned}
M_{0}: L_{p}\left(\mathbb{R}_{+} ; \dot{H}_{p}^{-5 / 4}\left(\mathbb{R}^{n}\right)\right. & \left.\cap \mathcal{S}_{R}^{\prime}\left(\mathbb{R}^{n}\right)\right) \\
\partial_{t} M_{0}: L_{p}\left(\mathbb{R}_{+} ; L_{p}\left(\mathbb{R}^{n}\right) \cap \mathcal{S}_{R}^{\prime}\left(\mathbb{R}_{+} ; H_{p}^{m}\right)\right) & \rightarrow L_{p}\left(\mathbb{R}_{+} ; H_{p}^{m}\left(\mathbb{R}^{n}\right) \cap \dot{H}_{p}^{-1 / 4}\left(\mathbb{R}^{n}\right)\right),
\end{aligned}
$$

as well as

$$
\begin{aligned}
M_{3}: L_{p}\left(\mathbb{R}_{+} ; \dot{H}_{p}^{-7 / 4}\left(\mathbb{R}^{n}\right) \cap \mathcal{S}_{R}^{\prime}\left(\mathbb{R}^{n}\right)\right) & \rightarrow L_{p}\left(\mathbb{R}_{+} ; H_{p}^{m}\left(\mathbb{R}^{n}\right)\right), \\
\partial_{t} M_{3}: L_{p}\left(\mathbb{R}_{+} ; \dot{H}_{p}^{-1 / 4}\left(\mathbb{R}^{n}\right) \cap \mathcal{S}_{R}^{\prime}\left(\mathbb{R}^{n}\right)\right) & \rightarrow L_{p}\left(\mathbb{R}_{+} ; H_{p}^{m}\left(\mathbb{R}^{n}\right)\right),
\end{aligned}
$$

are bounded, for all $m \in \mathbb{N}$.

Finally we consider the one-phase case. As shown in Section 5, then we have the asymptotics

$$
\lambda_{j}(|\xi|) \sim \pm i|\xi|^{3 / 2} \sqrt{\sigma / \rho}-2 \mu|\xi|^{2} / \rho,
$$

hence

$$
m_{j}(z,|\xi|) \sim \frac{1}{2} \frac{1}{\lambda+2 \mu|\xi|^{2} / \rho \mp i|\xi|^{3 / 2} \sqrt{\mu / \rho}} .
$$

This implies that $M_{3}$ maps $L_{p}\left(\mathbb{R}_{+} ; \dot{H}_{p}^{-2}\left(\mathbb{R}^{n}\right) \cap \mathcal{S}_{R}^{\prime}\left(\mathbb{R}^{n}\right)\right)$ into $L_{p}\left(\mathbb{R}_{+} ; H_{p}^{m}\left(\mathbb{R}^{n}\right)\right)$, and $\partial_{t} M_{3}$ maps accordingly $L_{p}\left(\mathbb{R}_{+} ; \dot{H}_{p}^{-1 / 2}\left(\mathbb{R}^{n}\right) \cap \mathcal{S}_{R}^{\prime}\left(\mathbb{R}^{n}\right)\right)$ into $L_{p}\left(\mathbb{R}_{+} ; H_{p}^{m}\left(\mathbb{R}^{n}\right)\right)$, for each $m \in \mathbb{N}$.

For the symbol $m_{0}(z, \tau)$ we obtain in this case the estimate

$$
\left|m_{0}(z, \tau)\right| \leq C a^{3} \int_{r_{0}}^{\infty} \frac{d r}{\left(r^{2}+a\right)^{2}(|z|+r) \sqrt{r}},
$$

since $\operatorname{Im} \Phi_{+}(r) \sim c / \sqrt{r}$ as $r \rightarrow \infty$. The scaling $r=\sqrt{a} s$ yields

$$
a^{3} \int_{r_{0}}^{\infty} \frac{d r}{\left(r^{2}+a\right)^{2}(|z|+r) \sqrt{r}}=a^{3 / 4} \int_{r_{0} / \sqrt{a}}^{\infty} \frac{d s}{\left(s^{2}+1\right)^{2} \sqrt{s}(s+|z| / \sqrt{a})} .
$$

The integral from 1 to $\infty$ is estimated by

$$
a^{3 / 4} \int_{1}^{\infty} \frac{d s}{\left(s^{2}+1\right)^{2} \sqrt{s}(s+|z| / \sqrt{a})} \leq c \frac{a^{5 / 4}}{|z|+a^{1 / 2}} \leq c \frac{|\xi|^{3 / 4}}{|\lambda|+|\xi|^{3 / 2}},
$$

and the corresponding integral from $r_{0} / \sqrt{a}$ by means of

$$
a^{3 / 4} \int_{r_{0} / \sqrt{a}}^{1} \frac{d s}{\left(s^{2}+1\right)^{2} \sqrt{s}(s+|z| / \sqrt{a})} \leq c \frac{a}{|z|+r_{0}}=c \frac{|\xi|}{|\lambda|+r_{0}|\xi|^{2}} .
$$

This estimate is optimal, up to constants. Thus for small frequencies we see that the operators

$$
\begin{aligned}
& M_{0}: L_{p}\left(\mathbb{R}_{+} ; \dot{H}_{p}^{-1}\left(\mathbb{R}^{n}\right) \cap \mathcal{S}_{R}^{\prime}\left(\mathbb{R}^{n}\right)\right) \rightarrow L_{p}\left(\mathbb{R}_{+} ; H_{p}^{m}\left(\mathbb{R}^{n}\right)\right), \\
& \partial_{t} M_{0}: L_{p}\left(\mathbb{R}_{+} ; L_{p}\left(\mathbb{R}^{n}\right) \cap \mathcal{S}_{R}^{\prime}\left(\mathbb{R}^{n}\right)\right) \rightarrow L_{p}\left(\mathbb{R}_{+} ; H_{p}^{m}\left(\mathbb{R}^{n}\right) \cap \dot{H}_{p}^{-3 / 4}\left(\mathbb{R}^{n}\right)\right),
\end{aligned}
$$

are bounded, for all $m \in \mathbb{N}$.

Acknowledgments. The authors would like to thank Prof. Yoshihiro Shibata and Prof. Senjo Shimizu for stimulating discussions on the subject of this paper. 
The research of GS was partially supported by NSF, Grant DMS-0600870. The second author wants to thank the Department of Mathematics, Vanderbilt University, Nashville, $\mathrm{TN}$, for its kind hospitality.

\section{References}

[1] G. Allain, Small-time existence for the Navier-Stokes equations with a free surface, Appl. Math. Optim. 16 (1987), 37-50.

[2] J. T. Beale, Large-time regularity of viscous surface waves, Arch. Rational Mech. Anal. $84(1983 / 84), 304-352$.

[3] D. Bothe and J. Prüss, $L_{p}$-theory for a class of non-Newtonian fluids, SIAM J. Math. Anal. 39 (2007), 379-421.

[4] I. V. Denisova, A priori estimates for the solution of the linear nonstationary problem connected with the motion of a drop in a liquid medium, Trudy Mat. Inst. Steklov 188 (1990), 3-21 (in Russian); translation in: Proc. Steklov. Inst. Math. 1991, no. 3, 1-24.

[5] I. V. Denisova, Problem of the motion of two viscous incompressible fluids separated by a closed free interface, in: Mathematical Problems for Navier-Stokes Equations (Centro, 1993), Acta Appl. Math. 37 (1994), 31-40.

[6] I. V. Denisova and V. A. Solonnikov, Classical solvability of the problem of the motion of two viscous incompressible fluids, Algebra i Analiz 7 (1995), no. 5, 101-142 (in Russian); translation in: St. Petersburg Math. J. 7 (1996), no. 5, 755-786.

[7] R. Denk, M. Hieber, and J. Prüss, $\mathcal{R}$-boundedness, Fourier multipliers, and problems of elliptic and parabolic type, Mem. AMS 788 (2003).

[8] R. Denk, M. Hieber, and J. Prüss, Optimal $L^{p}-L^{q}$-estimates for parabolic boundary value problems with inhomogeneous data, Math. Z. 257 (2007), 193-224.

[9] W. Desch, M. Hieber, and J. Prüss, $L_{p}$-theory of the Stokes equation in a half space, J. Evol. Equ. 1 (2001), 115-142.

[10] G. Dore and A. Venni, On the closedness of the sum of two closed operators, Math. Z. 196 (1987), 189-201.

[11] M. Hieber and J. Prüss, Functional calculi for linear operators in vector-valued $L^{p}$-spaces via the transference principle, Adv. Differential Equations 3 (1998), 847-872.

[12] J. Prüss and G. Simonett, $H^{\infty}$-calculus for the sum of noncommuting operators, Trans. Amer. Math. Soc. 359 (2007), 3549-3565.

[13] J. Prüss and G. Simonett, On the two-phase Navier-Stokes equations with surface tension, submitted.

[14] J. Prüss and H. Sohr, On operators with bounded imaginary powers in Banach spaces, Math. Z. 203 (1990), 429-452.

[15] Y. Shibata and S. Shimizu, On a free boundary value problem for the Navier-Stokes equations, Differential Integral Equations 20 (2007), 241-276.

[16] Y. Shibata and S. Shimizu, Free boundary value problems for a viscous incompressible fluid, in: Kyoto Conference on the Navier-Stokes Equations and their Applications, RIMS Kokyuroku Bessatsu, B1, Res. Inst. Math. Sci., Kyoto, 2007, 356-358.

[17] V. A. Solonnikov, Solvability of the problem of evolution of an isolated amount of a viscous incompressible capillary fluid, in: Mathematical Questions in the Theory of Wave Propagation, 14, Zap. Nauchn. Sem. Leningrad. Otdel. Mat. Inst. Steklov. (LOMI) 140 (1984), 179-186 (in Russian); translation in: J. Soviet Math. 37 (1987). 
[18] V. A. Solonnikov, Unsteady motions of a finite isolated mass of a self-gravitating fluid, Algebra i Analiz 1 (1989), no. 1, 207-249 (in Russian); translation in: Leningrad Math. J. 1 (1990), no. 1, 227-276.

[19] V. A. Solonnikov, An initial-boundary value problem for a Stokes system that arises in the study of a problem with a free boundary, Trudy Mat. Inst. Steklov. 188 (1990), 150-188 (in Russian); translation in: Proc. Steklov Inst. Math. 1991, no. 3, 191-239.

[20] V. A. Solonnikov, Solvability of a problem on the evolution of a viscous incompressible fluid, bounded by a free surface, on a finite time interval, Algebra i Analiz 3 (1991), no. 1, 222-257 (in Russian); translation in: St. Petersburg Math. J. 3 (1992), no. 1, 189-220.

[21] V. A. Solonnikov, $L_{q}$-estimates for a solution to the problem about the evolution of an isolated amount of a fluid, J. Math. Sci. (N. Y.) 117 (2003), 4237-4259.

[22] N. Tanaka, Two-phase free boundary problem for viscous incompressible thermo-capillary convection, Japan J. Mech. 21 (1995), 1-41.

[23] A. Tani, Small-time existence for the three-dimensional Navier-Stokes equations for an incompressible fluid with a free surface, Arch. Rat. Mech. Anal. 133 (1996), 299-331.

[24] A. Tani and N. Tanaka, Large-time existence of surface waves in incompressible viscous fluids with or without surface tension, Arch. Rat. Mech. Anal. 130 (1995), 303-304. 
